\title{
'Cognitive strategy' in visual search: how it works and when it generalises
}

\author{
David J. Yates \& Tom Stafford \\ Department of Psychology, University of Sheffield
}

\begin{abstract}
Recent evidence suggests that participants perform better on some visual search tasks when they are instructed to search the display passively (i.e. letting the unique item "pop" into mind) rather than actively (Smilek, Enns, Eastwood, \& Merikle, 2006; Watson, Brennan, Kingstone, $\&$ Enns, 2010). We extended these findings using eye tracking, a neutral baseline condition (Experiment 1$)$ and testing visual search over a wider range of eccentricies $\left(10^{\circ}-30^{\circ}\right.$, Experiment 2). We show that the passive instructions led to participants delaying their initial saccade compared to participants given active or neutral instructions. Despite taking longer to start searching the display, passive participants then find and respond to the target faster. We show that this benefit does not extend to search where items were distributed in the true periphery.
\end{abstract}

UNPUBLISHED MANUSCRIPT FROM 2013-14: After initial review at a journal additional experimental work was requested, which we were not in a position to carry out. From DY's letter to the editor withdrawing the manuscript:

The major concern of yourself and the reviewers was data loss in the eye movement results. Having reviewed this, we agree this is a substantial issue. As Reviewer 1 pointed out, most of the dropped trials (34.2\% in Experiment 1 and $30.4 \%$ in Experiment 2) were the result of anticipatory saccades at the beginning of the trials. This is likely because of the fixed trial timing with respect to the fixation point, a procedure we employed because we based our work on that of Smilek and colleagues (2006). We have therefore come to the conclusion that the only way we could significantly reduce the number of dropped trials in the eye movement results would be to re-run the experiments with 1) variable onset timing to reduce the number of anticipatory saccades and 2) an eye tracker that has higher spatial and temporal resolution, as Reviewer 2 suggested.

Although we feel that the behavioural results of the visual search experiment still hold (in particular the comparison to the neutral condition is novel), we are regretfully convinced that we are not in a position to allay these concerns.

\section{Introduction}

Whether we are scanning our desk for a pen, or the forest shadows for the eyes of a predator, we are engaging in visual search. The study of visual search gives us an opportunity to experimentally inspect the operation of automatic perceptual processing in the service of purposive action, specifically that of locating a target. Most visual search studies either vary some characteristic of the display, such as the similar-

David Yates was funded by a White Rose University Consortium Doctoral Training network. We thank the other members of the network for advice and support: Kevin Gurney, Jim Austin, Simon O'Keefe, Marc de Kamps, Netta Cohen, Becky Naylor and Dave Harrison. Ben Tatler, Megan Freeth and Alex Cope provided valuable advice. ity between target and distractors, or the prior knowledge or expectations that are brought to the task by the participant.

In 2006, Smilek and colleagues published results which showed that visual search could be enhanced by a simple change in general strategy. They noted anecdotal evidence from experienced visual search researchers which suggested that a passive, or more relaxed, strategy could lead to more efficient search. They formally demonstrate this effect using experiments in which participants searched for a circle with a gap on either the left or right hand side amongst distractors with a gap on both sides. Easy and hard versions of the task were tested, which was achieved by changing the size of the gap in the target and so the difficulty of the discrimination of the target from the distractors. Two strategy conditions were tested, with the only thing that varied between them being the instructions that were given to participants. Half the participants were asked "to be as receptive as possible, and let the 
unique item "pop" into your mind" ("passive' instructions). The other half were asked "to be as active as possible and "search" for the item" ('active' instructions). The passive group performed significantly better than the active group in the hard version of the visual search task.

This result seems to offer a quick and easy way of improving an important cognitive function. Smilek and colleagues suggested that the effect could be due to the passive instructions giving fast automatic processes more influence over spatial attention and active instructions leading to a greater reliance on slow and unnecessary executive control processes. A complementary perspective can be had by reviewing work on eye-movements in visual search.

Hooge and Erkelens (1999) investigated the relationship between fixation duration, peripheral analysis and discrimination in a visual search task that used similar stimuli to the Smilek experiments. Their experiment manipulated the discrimination and selection tasks independently from one another. They found that fixation duration was affected by the discrimination task only: participants would (unsurprisingly) fixate for longer if the target and distractor were very similar to one another and so the discrimination task was difficult. So, by manipulating the difficulty of the discrimination task, Hooge and Erkelens could manipulate fixation duration and then assess the quality of the participants' subsequent eye movements (i.e. did they fall on items that were more like the target). What they found was clear: by increasing the difficulty of the discrimination task, and so the fixation duration, participants made better subsequent eye movements and searched more efficiently.

Watson, Brennan, Kingstone and Enns (2010), conducted a similar visual search experiment to Smilek, with the addition of eye tracking throughout. Their study showed that the instructions used in the Smilek experiments altered eyemovements, which in turn allowed more efficient information processing and search. They found that passive instructions led to faster response times (RTs) overall, a shallower search function and more errors than the active group. They combined RT and number of errors to form an inefficiency score and showed that the passive group were still significantly faster than the active group, even when this method of accounting for lower accuracy was used.

Watson's experiment shows that the strategy instructions led to systematic differences in the way the participants searched the display. Watson goes on to argue that their results show that the passive instructions lead to an emphasis on seeing and the active to an emphasis on looking. Specifically, the passive participants are able to use the extra information they gain from their longer initial fixation to help guide their search, and so require fewer and more direct saccades to the target.

\section{The present experiments}

Together these studies (Smilek et al., 2006; Watson et al., 2010) offer the promise of a simple instruction which can significantly enhance visual search. This promise, however, is accompanied by something of a conundrum. That visual search performance can be improved by a general injunction to relax is surprising for two reasons. Firstly, because in most domains we expect that performance improves when we try harder, rather than when we try less hard. Secondly, the result raises the question of why visual search performance has this potential to be so easily improved. Visual search is a fundamental cognitive function, something which we might expect to have been under selection pressure throughout both our evolutionary history, and over our individual lifespans, across a wide range of domains. Should we believe that most people, most of the time, adopt a suboptimal visual search strategy?

The present research addresses this conundrum. The first experiment addresses the question of whether the passive instructions aid visual search, or whether the active instructions hinder it. Neither the research by Smilek et al. (2006) or Watson et al. (2010) used a neutral condition to contrast the effects of the active and passive strategies. Given this, it is not possible to say whether the passive instructions improved search efficiency, the active instruction made search efficiency worse, or a combination of the two.

The second experiment addresses the question of whether the advantage of passive instructions holds when stimuli are distributed over the full visual field. Eye-movements occur because only a small central area of the visual field, the fovea, supports high resolution perception (Findlay \& Gilchrist, 2003). The majority of studies of visual perception, including those of visual search, are conducted using stimuli that fall on or near the fovea (i.e. within $+/-10^{\circ} \mathrm{ec}-$ centricity). Visual function extends far beyond this range, albeit with changes in resolution and response time (Yates \& Stafford, 2011). Our perspective is to study visual function within the context of full field stimuli. This is the context in which visual search is optimised in evolutionary and ontogenetic time.

\section{Experiment 1}

\section{Method}

This experiment is a replication of Experiment 1 by Smilek et al., (2006) with the addition of a third group of participants who were given neutral instructions and eye tracking.

Participants. 42 participants aged between 16 and 31 ( $M=20.0$ years, $S D=3.0$ years) took part in the experiment (35 Female, 7 Male). 35 of the participants took part in return for course-credit towards their undergraduate Psychology degrees. The participants were assigned to the instruc- 
tional conditions so that there was an even number (14) in each group. However, three participants were removed from the eye movement analysis because their eye movement data were not reliable, leaving 13 in each instructional group.

Apparatus and Stimuli. This visual search task involved participants searching for a target circle, which had a gap on either the left or right hand side, amongst distractor circles that had a gap on both sides. In each trial the target and either one, three or five distractors were arranged around an invisible ring with a radius of $4^{\circ}$. The locations for the items were chosen at random on each trial from eight possible fixed locations, spaced evenly around the ring at $45^{\circ}$ angles.

The targets and distractors were $0.8^{\circ}$ in diameter. The gaps on the left and right of the distractors were always $0.1^{\circ}$. The gaps in the target circles varied depending on the difficulty of the task. In the easy condition, the gap in the target was $0.3^{\circ}$ and in the hard condition the gap in the target was $0.15^{\circ}$.

Stimuli were presented on a Sony SDM-HS95P Monitor at a resolution of $1280 \times 1024$ pixels with a refresh rate of $60 \mathrm{~Hz}$. The experiment was written in Matlab using the Psychophysics Toolbox extensions (Brainard, 1997; Pelli, 1997; Kleiner, Brainard, Pelli, Ingling, \& Murray, 2007). The monitor was secured at a viewing distance of $57 \mathrm{~cm}$, meaning 1 $\mathrm{cm}$ subtended $1^{\circ}$ visual angle. Head movements were restricted during the experiment using a custom-built, heightadjustable chin rest. The centre of the left pupil of the participant was tracked using a head-mounted ISCAN RK-500 at a temporal resolution of $60 \mathrm{~Hz}$.

Design and Procedure. Following 12 practice trials, the participant undertook two blocks of trials with a break in between: 144 easy trials and 144 hard trials (the order of presentation was counterbalanced between participants). Within each block there were three set sizes (2, 4 or 6) and two types of target (left-target or right-target). Each of the stimuli combinations were repeated 24 times in a random order.

Before each block of trials the participant was first read one of three sets of strategy instructions: passive instructions, active instructions or neutral instructions (see below). The participant then undertook a standard nine-point calibration of the eye tracker and started the block of trials.

Each trial consisted of a fixation cross at the centre of the screen for $500 \mathrm{~ms}$, followed by a blank screen for $400 \mathrm{~ms}$, followed by a search display. The search display would stay on screen until the participant made a response or for a maximum of $1800 \mathrm{~ms}$. The "z" and "/" keys on the keyboard had been marked with a white sticker with an " $L$ " and " $R$ " on them respectively. The participants rested their index fingers over these keys and were instructed to respond as quickly as possible without sacrificing accuracy for speed. Participants were given feedback (correct / incorrect / time out) after each trial.
Passive Instructions. "The best strategy for this task, and the one that we want you to use in this study, is to be as receptive as possible and let the unique item "pop" into your mind as you look at the screen. The idea is to let the display and your intuitions determine your response. Sometimes people find it difficult or strange to tune in to their "gut feeling" - but we would like you to try your best. Try to respond as quickly and accurately as you can while using this strategy. Remember, it is very critical for this experiment that you let the unique item just "pop" into your mind."

Active Instructions. "The best strategy for this task, and the one that we want you to use in this study, is to be as active as possible and to "search" for the item as you look at the screen. The idea is to deliberately direct your attention to determine your response. Sometimes people find it difficult or strange to "direct their attention" - but we would like you to try your best. Try to respond as quickly and accurately as you can while using this strategy. Remember, it is very critical for this experiment that you actively search for the unique item."

Neutral Instructions. "The best strategy for this task, and the one that we want you to use in this study, is to respond as quickly and accurately as you can."

\section{Measures.}

Visual Search Performance. The mean correct RTs and number of errors were recorded for each participant in each condition. These two measures were then combined into a single inefficiency score, which is calculated as the mean correct RT / proportion correct for each participant in each condition.

Eye Movement Metrics. The data were first split in to fixations and saccades using a simple velocity threshold algorithm. In both experiments, the velocity threshold used was $60^{\circ} / \mathrm{sec}$ (equivalent to $1 \%$ sample). After detection of a saccade, the algorithm then searched for the onset and offset of that particular saccade using a threshold of $25^{\circ} / \mathrm{sec}$.

Fixation duration is of particular interest in this experiment. However, there are a number of studies that show significant differences between the initial fixation duration (after display onset) and subsequent, mid-trial fixations (Motter \& Belky, 1998b, 1998a; Hooge \& Erkelens, 1999; Zelinsky \& Sheinberg, 1997). For example, Zelinsky and Sheinberg (1997) and Hooge and Erkelens (1999), both found that apparent differences in fixation duration during search are sometimes based entirely on the first fixation. With this simple requirement in mind, each trial was analysed using the following metrics:

Initial fixation duration. This is defined as the time between the onset of the search display and the participant making their first saccade. A trial was only included in the eye movement analysis if the participant was fixated within a $3^{\circ}$ central region for at least $50 \mathrm{~ms}$ at the start of the trial. In total, across all conditions, $34.2 \%$ of trials were removed from 
eye movement analysis for not meeting this criterion. Although this figure varied between participants, there was no significant differences between the number of trials excluded from analysis by strategy group $(F(2,33)=0.01, p=.989)$.

Overall search duration. The time between the end of the first saccade and the start of the first fixation in the target region, which was defined as a $3^{\circ}$ circle centred on the target. An additional $10.0 \%$ of trials were excluded from further analysis because the participant did not fixate the target region during the trial. Again, there was no difference in the number of trials excluded between strategy groups $(F(2,33)=0.92, p=.407)$.

Average search fixation duration. This was defined as the search duration divided by the number of fixations made during this time.

Time to respond. The time between the start of the first fixation in the target region and the participant making a button press response.

Initial fixation duration versus search and response time. For each trial, the time spent searching and responding to the target was calculated by subtracting the initial fixation duration from the overall reaction time. We then plotted this value against the initial fixation duration for each trial to assertain whether there was any relationship between the time taken to start searching the display and subsequent success on the trial; in other words, did taking longer on the initial fixation lead to a faster response time in this particular task, regardless of condition? A linear regression was performed on each participants' plot and the linear coefficients were then averaged for each strategy group.

Statistical Tests. The visual search and eye movement results were analysed by a mixed analysis of variance (ANOVA) that assessed the within-participant factors of search difficulty (easy, hard) and set size $(2,4,6)$ and the between-participant factors of strategy group (neutral, passive, active) and order (easy first, hard first). A multiple comparisons post-hoc test with a Bonferroni correction was performed to determine any significant differences between the three strategy groups.

\section{Results}

Visual Search.

Reaction Times. The mean correct RTs increased with set size $(F(2,72)=646.22, p<.001)$ and the slope of these search functions were steeper when the task was more difficult $(F(2,72)=62.75, p<.001)$, indicating that the difficulty manipulation worked. More importantly, there was a significant main effect of the strategy instructions $(F(2,36)=$ $5.89, p=.006)$, with passive instructions leading to significantly faster performance than active instructions $(p=.006)$. Passive participants were also faster than neutral participants, but this was only approaching significance $(p=.076)$. However, unlike the results of Smilek et al., (2006), there was no significant interaction with set size $(F(4,72)=1.79$, $p=.141)$, indicating that passive participants were faster at searching but there was no significant change to the slope of their search function.

The easy and hard data were analysed separately in order to look at the effect of the instructions in more detail. This revealed that instructions had a substantial effect on the speed of search when search was hard $(F(2,36)=8.59, p=.001)$, but the effect of instructions was only approaching significance in the easy condition $(F(2,36)=3.11, p=.057)$. In the hard condition, the passive group were significantly faster than either the active $(p=.001)$ or neutral $(p=.010)$ groups and there was no difference between the active and neutral groups $(p=1)$. In the easy condition the passive group were faster than the active group ( $p=.053)$, but there was little or no difference between neutral and passive $(p=.508)$ or neutral and active $(p=.857)$ groups.

There was also a significant effect of order of difficulty in the hard condition $(F(1,36)=9.36, p=.004)$ but not the easy condition $(F(1,36)=0.40, p=.533)$. This indicates that the participants performed significantly better on the hard task if they completed it after the easy task rather than before. However, this effect did not interact with set size or instructions.

Errors. As would be expected in a visual search task, the number of errors increased when there were more items in the display to search $(F(2,72)=50.09, p<.001)$ and when the task was more difficult $(F(2,72)=56.73, p<.001)$. However, the strategy instructions made no difference to the number of errors made $(F(2,36)=0.89, p=.419)$, and this was equally true for the easy $(F(2,36)=0.43, p=.657)$ and hard $(F(2,36)=0.94, p=.402)$ conditions.

Inefficiency Scores. Figure 1 shows that the inefficiency scores followed a very similar pattern of results as the mean correct RTs. There was a significant effect of strategy instructions overall $(F(2,36)=3.86, p=.030)$, and this effect was much more substantial in the hard condition $(F(2,36)=$ $4.57, p=.017$ ) where the passive groups were significantly faster than both the active $(p=.038)$ and neutral $(p=.039)$ groups. There was again no difference between neutral and active groups $(p=1)$.

Eye Movements.

Initial fixation duration. Participants took longer to start searching the display when there were more items to search $(F(2,66)=68.73, p<.001)$, but were faster to start searching overall in the hard block of trials $(F(1,33)=9.73$, $p=.004)$. The ANOVA and post-hoc tests revealed a significant main effect of instructions on the initial fixation duration $(F(2,33)=5.82, p=.007)$, with the passive group taking longer to start searching the display than either the active $(p=.009)$ or neutral $(p=.022)$ groups. The instructions had a stronger impact in the easy condition $(F(2,33)=6.12, p=.005)$, with the passive group taking longer to start searching than either the active $(p=.009)$ 


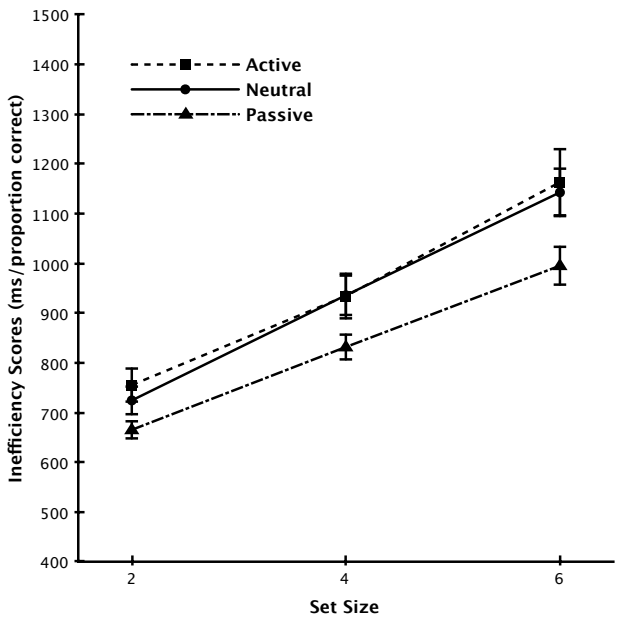

Figure 1. Experiment 1: Inefficiency scores (collapsed across easy and hard conditions). The error bars represent one standard error of the mean.

or neutral groups ( $p=.023$ ). Although the same pattern of results can be seen in the hard condition, the effect is not significant $(F(2,33)=2.35, p=.111)$.

Overall search duration. The overall search duration followed a very similar pattern as the mean correct RTs, with participants taking longer to find the target when there were more items to search $(F(2,66)=330.06, p<.001)$ and when the task was more difficult $(F(1,33)=143.06, p<.001)$. As with the mean correct RT, the instructions also had a significant effect $(F(2,33)=6.23, p=.005)$. Despite taking longer to start searching the display, the passive group found the target significantly faster than either active $(p=.007)$ or neutral groups $(p=.038)$. The effect of instructions was more substantial in the hard condition $(F(2,33)=4.97, p=.013)$ than in the easy condition $(F(2,33)=3.24, p=.052)$.

Average search fixation duration. The participants spent significantly longer on fixations when searching for a hard target $(F(1,33)=172.91, p<.001)$. However, the number of items in the display $(F(2,66)=0.68, p=.509)$ and strategy instructions $(F(2,66)=0.03, p=.973)$ had no effect on search fixation duration. This was equally true in the easy and hard conditions.

Time to respond. There was also a significant effect of instructions on how long it took the participants to make a button press once the target had been located $(F(2,33)=$ $5.12, p=.012$ ). Here, the passive group were significantly faster to make a button press compared to the active group $(p=.012)$. Although the passive group were also faster than the neutral group, this difference was not significant $(p=.166)$, nor was the difference between the active and neutral groups $(p=.818)$.

Initial fixation duration versus search and response time. We also found a relationship between the initial fixation du- ration and the subsequent response time. In the easy condition, the longer the participants took to start searching the display, the faster they were to find and respond to the target. One-sample t-tests revealed that the slopes of the neutral $(t(12)=-4.15, p=.001)$, passive $(t(12)=-7.28$, $p<.001)$ and active $(t(12)=-4.67, p=.001)$ conditions were all different from zero in the easy condition. A one-way ANOVA revealed that there was no significant difference between the slopes of the three conditions $(F(2,38)=0.70$, $p=.505)$. In the hard condition one-sample t-tests revealed that the slopes of the neutral $(t(12)=-0.88, p=.397)$, passive $(t(12)=-1.13, p=.279)$ and active $(t(12)=-0.29$, $p=.778)$ conditions were not significantly different from zero and a one-way ANOVA revealed that there was no significant difference between the three slopes $(F(2,38)=0.06$, $p=.949)$.

\section{Discussion}

The results of this experiment confirm the general finding of both Smilek et al., (2006) and Watson et al., (2010): in this particular task, giving participants passive instructions leads to significantly faster search times compared to giving participants active instructions. However, by including a baseline, neutral instructions condition, this experiment extends these findings by showing that 1) the active group were statistically indistinguishable from the baseline and 2) the passive group were significantly faster than the baseline. This suggests that passive instructions genuinely improve performance, rather than the active instructions making performance worse.

There are a number of differences between the results reported here and Smilek's results that are worth mentioning given this is a replication. The first is that the participants in this experiment found the task more difficult overall: they were slower to respond and made more mistakes in both the easy and hard conditions. This may have been caused by the inclusion of the head-mounted eye tracker, which partically obscures the line of sight, or other low-level factors that could not be exactly replicated. Whatever the cause of this difference, the interesting side-effect is that we have four levels of difficulty to compare. So in Smilek's easy condition, there was no difference between the mean correct RTs in the passive and active groups in the easy condition $(F<1)$ and yet in our easy condition the difference was approaching significance $(p=.053)$. This just-approaching-significant difference between the passive and active group suggests that the passive versus active advantage scales in proportion to the difficulty of the task.

The second difference is that the passive instructions also led to a significantly shallower search function in Smilek's experiment $(p<.003)$ and Watson's experiment $(p<.05)$, but this interaction between set size and instructions was not significant in this experiment $(p=.141)$. This suggests that the passive instructions in Smilek and Watson's studies led to 
more efficient search, but in my experiment they only made search times faster. However, Smilek and Watson also report that the passive group made more errors than the active group, implying that there was some trade-off occurring between speed and accuracy. However, this was not the case in this experiment: the strategy instructions had no effect on the number of mistakes made. This difference in strategy could account for the change in search functions found in Smilek and Watson's studies: if the passive participants are trading speed for accuracy, this would result in a shallower search function in the passive group. The search efficiency scores correct most of this difference, and it is clear that in Watson's experiment at least, doing so has reduced the interaction between set size and instructions from $p<.05$. to $p<.07$, whereas in my experiment, taking the errors into account has increased the significance of the interaction between set size and instructions from $p=.141$ to $p=.070$. It is not clear whether the search efficiency scores reduced this interaction in Smilek's experiment as he just reports that efficiency scores showed the same pattern of results as the correct RT data.

The eye movement analysis revealed a similar pattern of results to those of Watson and colleagues (2010). Specifically, both experiments found that the passive participants took longer to start searching the display, found the target faster and were faster to make a response once the target was found. However, the baseline, neutral instructions condition allow us to extend the findings of Watson and colleagues by showing that: 1) active participants were statistically indistinguishable from the neutral participants in every measure of eye movements and 2) the passive instructions led to a significant change in the way the participants searched the display. This pattern of results exactly mirrors that found in the visual search results.

Finally, the eye movement analysis revealed a significant advantage of spending longer on the initial fixation regardless of the instructional group participants were in. For every millisecond extra that the participant took to start searching, the participant would find the target and respond around 0.83 $\mathrm{ms}$ faster on average across all conditions. This indicates that, in line with the findings of Hooge and Erkelens (1999), taking longer on the initial fixation permits better peripheral analysis, which results in better eye movements (i.e. eye movements that are more likely to find the target). Furthermore, once the target has been located, the longer initial fixation and peripheral analysis means that they are more likely to have started to identify whether the target is left or right sided, so are faster to discriminate the target once it has been fixated and make a button press response.

The results of this experiment suggest that the participants performed sub-optimally on this task by default, and were made to improve by simply being instructed to search passively before hand. Furthermore, it is clear that, in this par- ticular task, it is a good strategy to spend longer on the initial fixation but participants do not adopt this strategy by default. However, giving participants passive instruction makes them adopt this strategy (or be biased towards it) and the result is that they find and respond to the unique item more quickly.

A corollory of this conclusion is that the passive advantage should not be found in a search task where the initial, central fixation does not allow effective peripheral analysis of the scene. In Smilek and Watson's experiments, where an advantage was seen, all the stimuli were either $4^{\circ}$ (Smilek) or between $1.75^{\circ}-7^{\circ}$ (Watson) from the initial fixation. This is within a reasonable range of eccentricities for effective peripheral analysis using the perifovea. If the targets are spaced further away from fixation (i.e. $>10^{\circ}$ ), this would reduce the contribution the initial peripheral analysis could make to the task, and so the passive versus active advantage should not be found.

\section{Experiment 2}

Experiment 2 is based on the standard display condition of Watson and colleagues (2010) so that a comparison can be made between the results. The main difference here is that the experiment has been scaled up in size: the targets and distractors were eight times larger than those used by Watson $\left(4^{\circ}\right.$ versus Watson's $\left.0.5^{\circ}\right)$, and the participants searched for the target over a wider range of eccentricities $\left(10^{\circ}, 20^{\circ}\right.$, $30^{\circ}$ versus Watson's $1.75^{\circ}, 3.5^{\circ}, 7^{\circ}$ ).

Our prediction is that the passive versus active advantage will not be present in this high-eccentricity version of the task. The passive instructions may still cause the participants to spend longer on their first fixation compared to the active participants. However, doing so may not lead to the advantage that was seen in Experiment 1.

\section{Method}

Participants. 24 participants aged between 20 and 44 ( $M=25.2$ years, $S D=6.4$ years) took part in the experiment (13 Female, 11 Male). Twelve of the participants were recruited using the University of Sheffield volunteers list and were paid $£ 5$ for taking part. The remaining twelve participants were postgraduates or post-docs recruited from the University of Sheffield Psychology Department.

Apparatus and Stimuli. As in Experiment 1, this visual search task involved participants searching for a target circle with a gap on either the left or right hand side amongst a number of distractor circles that had a gap on both sides. In each trial the target and two, five or eight distractors were arranged around three invisible rings with radii of $10^{\circ}, 20^{\circ}$ and $30^{\circ}$. In each trial a third of the items were randomly placed on each of the three rings so that they could be no closer than 45 degrees of arc to each other. All items were the same size $\left(4^{\circ}\right.$ in diameter with gaps of $\left.1^{\circ}\right)$. See Figure 2 for an example display). 
Stimuli were presented on an immersive dome display, which allows images to be presented to participants over a range that extends $240^{\circ}$ in the horizontal axis and approximately $100^{\circ}$ in the vertical axis. This was achieved using a Canon XEED SX6 projector, which projects the $1400 \times 1050$ pixel image on to a curved mirror that reflects the image over the surface of the dome. This meant that all the items were white on a black background, rather than the black items on a white background used in Experiment 2. The centre of the left pupil of the participant was tracked using the same eye tracker used in Experiment 1.

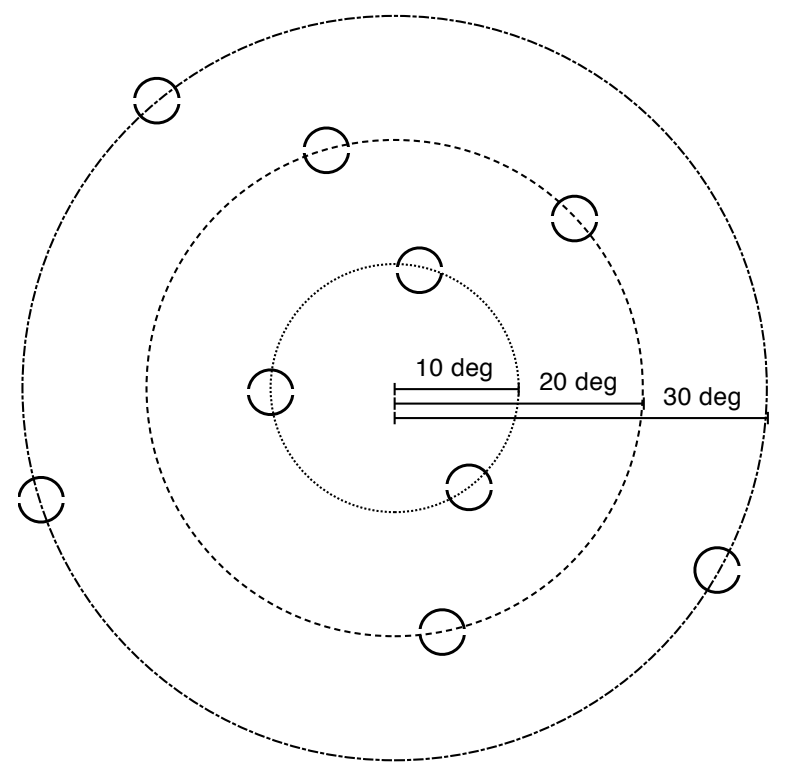

Figure 2. An illustration of the display used in Experiment 2 (to scale). In each trial, a third of the items were presented at each of the three eccentricities.

Design and Procedure. The participant undertook four blocks of 90 trials and was given breaks between each block. Within each block there were three set sizes $(3,6$ or 9$)$, three target eccentricities (target displayed in $10^{\circ}, 20^{\circ}$ or $30^{\circ}$ ring) and two types of target (left-target or right-target). Each of the 18 stimuli combinations was repeated 20 times in a random order.

Before each block of trials the participant was first read one of two sets of strategy instructions: passive instructions or active instructions. The participant then undertook a standard nine-point calibration of the eye tracker and started the block of trials.

Each trial consisted of a fixation cross at the centre of the screen for $500 \mathrm{~ms}$, followed by a blank screen for $400 \mathrm{~ms}$, followed by a search display. The search display would stay on screen until the participant made a response or for a maximum of $3600 \mathrm{~ms}$. The "z" and "/" keys on the keyboard had been marked with a white sticker with an "L" and "R" on them respectively. The participants rested their index fingers over these keys and were instructed to respond as quickly as possible without sacrificing accuracy for speed. Participants were given feedback (correct / incorrect / time out) after each trial.

Eye Movement Metrics. The eye movement data were analysed in the same way as in Experiment 1. However, due to the larger stimuli used in Experiment 2, the central and target regions used were $7.5^{\circ}$ in diameter. As in Experiment 2 , a trial was only included in the eye movement analysis if the participant was fixated on the centre of the display (i.e. within the central region) for at least $50 \mathrm{~ms}$ at the start of each trial. In total, across all conditions, 30.4\% of trials were removed from eye movement analysis for not meeting this criterion. Although this figure varied between participants, there was no significant differences between the number of trials excluded from analysis by strategy group $(F(1,20)=0.12, p=.736)$. An additional $25.72 \%$ of trials were excluded from further analysis because the participant did not fixate the target region during the trial. Again, this number did not vary by strategy group $(F(1,20)=0.04$, $p=.839$ ).

\section{Results}

\section{Visual Search.}

Reaction Times. The participants took longer to respond when the target was further away from the centre of the display $(F(2,44)=198.54, p<.001)$ and when there were more items in the display to search $(F(2,44)=489.95$, $p<.001)$. There was also a significant interaction between target eccentricity and set size $(F(4,88)=13.71, p<.001)$, indicating that the search functions were steeper when the target was further from the centre of the display. However, unlike Experiment 1, there was no effect of instructions on the mean correct RTs $(F(1,22)=0.03, p=.873)$.

Errors. As well as being slower to respond, the participants also made significantly more mistakes when the target was further from the centre of the display $(F(2,44)=$ $3.36, p=.044)$ and when there were more items to search $(F(2,44)=23.36, p<.001)$. Similar to Experiment 1 , the strategy instructions did not change the number of errors made $(F(1,22)=0.02, p=.891)$.

Inefficiency Scores. The inefficiency scores showed the same pattern of results as the mean correct RT. The participants in Experiment 2 were affected by target eccentricity $(F(2,44)=127.91, p<.001)$ and set size $(F(2,44)=$ $324.86, p<.001)$, but not by the instructions that were given before hand $(F(1,22)=0.01, p=.915)$.

\section{Eye Movements.}

Initial fixation duration. Participants took longer to start searching the display when there were more items to search $(F(2,40)=61.80, p<.001)$. There was no significant main effect of strategy instructions on the time taken to start searching $(F(2,40)=0.19, p=.666)$. 


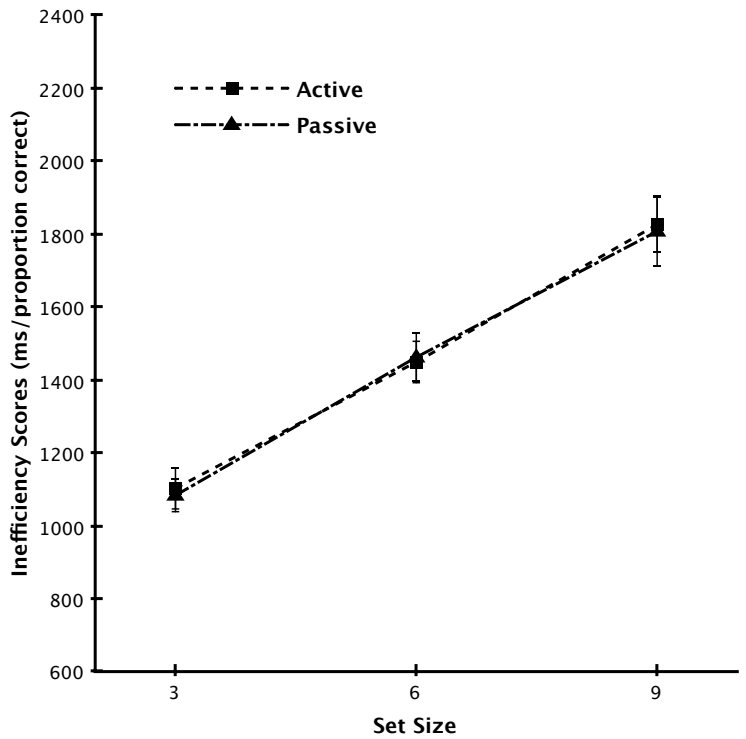

Figure 3. Experiment 2: Inefficiency scores. The error bars represent one standard error of the mean.

Search duration. Mirroring the mean correct RT results, participants took longer to find the target when the target was further from fixation $(F(2,40)=265.83, p<.001)$ and when there were more items to search $(F(2,40)=357.88$, $p<.001)$, and the search functions were steeper when the target was further away $(F(4,80)=9.97, p<.001)$. Strategy instructions had no effect on the search duration $(F(4,80)=0.27, p=.611)$.

Search fixation duration. Similar to the initial fixation duration, participants spent longer on their search fixations when there were more items to search $(F(2,40)=31.74$, $p<.001)$. The strategy instructions, however, had no effect on the search fixation durations $(F(2,40)=0.00, p=.962)$.

Time to respond. Participants were faster to respond to the target when the target was further from fixation $(F(2,40)=18.46, p<.001)$. However, there was no significant main effect of instruction $(F(2,40)=0.02, p=.889)$ on the response time.

Initial fixation duration versus search and response time. As with Experiment 1, we analysed the relationship between the initial fixation duration and subsequent search and response time. One-sample t-tests revealed that the slope of the regression for both the passive $(t(10)=0.01, p=.992)$ and active $(t(10)=-0.10, p=.925)$ groups were not significantly different from zero, indicating that the time it took participants to start searching had no impact on the remaining search and response time. An independent samples t-test also revealed that there was no significant difference between the slope of the passive and active groups $(t(20)=0.09$, $p=.933)$.

\section{Discussion}

The results of Experiment 2 are clear: there was no difference between the passive and active groups in this high eccentricity version of the visual search task. The mean correct RTs, search functions, number of errors made and inefficiency scores for the passive and active groups are statistically indistinguishable from one another, and this is true regardless of which of the three eccentricities the target was presented at. It would seem that, as predicted, the passive versus active advantage is lost when a wider range of eccentricities need to be searched.

Mirroring the visual search results, it is also clear that the instructions made no difference to the way the participants searched the display with their eyes during each trial. There was no significant differences between the passive and active groups in any of the eye movement metrics recorded. Most notably, the passive participants did not take longer to start searching the display than the active participants. Unlike Experiment 1 , there was no advantage to taking longer to start searching the display in terms of subsequent success on the trial. This suggests that by placing the items outside of the range that can be successfully analysed by the perifovea, the advantage of spending longer on the initial fixation has been lost. As a result of this, the passive versus active advantage, which seems reliant on this strategy, also disappears.

\section{General Discussion}

These results confirm the existence of a 'passive advantage' in visual search, but extend our knowledge of how and when it operates. Experiment 1 demonstrated that the instruction to perform visual search by relaxing and letting the answer "pop in to your mind" does truly improve performance relative to a neutral baseline or active search instructions (which led to indistinguishable levels of performance). Eye-tracking confirmed that the passive instructions shifted searchers' behaviour away from the neutral/active seachers'. Participants in the passive condition performed a longer initial fixation, which was associated with more efficient search. Additionally they were also quicker to respond to the target once they located it. Experiment 2 demonstrates that the passive advantage does not extend to displays which cover a wide field (up to $30^{\circ}$ in this case). The average differences between the passive and active groups are lost, both in terms of visual search performance and eye-movement characteristics.

The passive versus active advantage has now been found in three separate studies, including the present one (in addition to Smilek et al., 2006; Watson et al., 2010). Given that our Experiment 1 shows that the passive instructions improve search performance over a baseline result, it is worth considering whether this improvement could be seen in realworld visual search tasks. For example, would it be possi- 
ble to instruct or train individuals to search in a passive way and so improve their overall performance? Although further research will be needed to answer this question, the visual search and eye movement results of the experiments reported here give some indication of a) which types of search task would see an improvement and b) what types of experiment should be performed next to establish the generalisability of this finding.

Experiment 1 revealed that there was a close relationship between the passive versus active advantage and the way the participants moved their eyes. The small change in initial fixation duration suggests that the effect would be drowned out in search tasks that require the coordinated effort of the eyes, head and body, such as searching a room. Recent evidence from Brennan, Watson, Kingstone and Enns (2011) suggests that this is indeed the case. In two experiments, participants were asked to search a large computer display (Experiment 1) or office (Experiment 2) for everyday objects like a set of keys or a mug, and were instructed to do so in a passive or active way beforehand. In both these search tasks they found that the participants given active instructions found the objects faster than the participants given passive instructions. This finding seems to conflict with the results of Experiment 2 , reported here, where no difference was found between the active and passive participants. There are two additional factors in Brennan's study that may explain this discrepancy. First, Brennan and colleagues only found an active advantage when the objects were harder to find, so if the search task was relatively easy in our experiment, we would not expect to see an active advantage. Second, Brennan and colleagues found that success in the office-based search task was associated with an increase in head movements. In our Experiment 2 head mvoements were restricted, which may have removed the active participants' scope for success.

It would seem that the passive advantage is lost in search tasks where a large area has to be searched, but this still leaves a number of visual search tasks that might see an advantage. For example, CCTV operators or baggage handlers perform relatively low-eccentricity, monitor-based search on a regular basis. However, the analysis of the initial fixation duration, and the discussion above, indicates that there is another criteria that must be met for the effect to be seen: there must be a sudden onset of the display in order for there to be a longer initial fixation. There are few, if any, real-world equivalents of the sudden onset of a search display (Tatler, Hayhoe, \& Land, 2011). It is possible that feature of experimental trials produces effects that are unlikely to be observed in everyday search behaviour. Given this conclusion, further work needs to establish whether the passive versus active advantage remains in low-eccentricity search of dynamic displays, such as searching video footage from CCTV cameras. We predict that under these circumstance the passive versus active advantage will be lost due to the lack of a sudden on- set in a dynamic display. If this is shown to be the case, it will strongly suggest that the passive versus active advantage does not generalise to non-computer, non-trial based search tasks.

That the passive instructions produce a benefit relative to a baseline of neutral instructions is impressive. It suggest that, on average, participants natural search behaviour is suboptimal (Boot, Becic, \& Kramer, 2009). This is surprising given the amount of practice that the typical person has with looking for things. You might expect that we would all be expert 'visual searchers'. Smilek et al's results suggest that we're not, since we can - by simple instruction - be improved in our performance. The results from Experiment 2 suggest a resolution of this seeming paradox. Visual search performance of the typical participant may be suboptimal for a typical visual search experiment, over a narrow range of ecentricities. In these conditions search strategy can be adjusted via instruction to better suit the search display. However, the same adjustments of strategy may not benefit more ecologically realistic visual search conditions. It is these wider eccentricity search displays for which visual search strategy is presumably optimised, and, correspondingly, requesting a strategy adjustment does not improve performance, nor does it have pervasive impact on search behaviour.

\section{References}

Boot, W. R., Becic, E., \& Kramer, A. F. (2009). Stable individual differences in search strategy? The effect of task demands and motivational factors on scanning strategy in visual search. Journal of Vision, 9(3), 7, $1-16$.

Brainard, D. (1997). The psychophysics toolbox. Spatial Vision, 10(4), 433-436.

Brennan, A. A., Watson, M. R., Kingstone, A., \& Enns, J. T. (2011, August). Person perception informs understanding of cognition during visual search. Attention, Perception, E Psychophysics, 73(6), 1672-1693.

Findlay, J. M., \& Gilchrist, I. D. (2003). Active Vision: The Psychology of Looking and Seeing. Oxford University Press, UK.

Hooge, I. T. C., \& Erkelens, C. J. (1999). Peripheral vision and oculomotor control during visual search. Vision Research, 39(8), 1567-1575.

Kleiner, M., Brainard, D., Pelli, D., Ingling, A., \& Murray, R. (2007). What's new in Psychtoolbox-3. In Perception 36 ECVP Abstract Supplement.

Motter, B. C., \& Belky, E. J. (1998a). The guidance of eye movements during active visual search. Vision Research, 38(12), 1805-1815.

Motter, B. C., \& Belky, E. J. (1998b). The zone of focal attention during active visual search. Vision Research, 38(7), 1007-1022. 
Pelli, D. G. (1997). The VideoToolbox software for visual psychophysics: transforming numbers into movies. Spatial Vision, 10(4), 437-442.

Smilek, D., Enns, J. T., Eastwood, J. D., \& Merikle, P. M. (2006). Relax! Cognitive strategy influences visual search. Visual Cognition, 14(4), 543-564.

Tatler, B. W., Hayhoe, M. M., \& Land, M. F. (2011). Eye guidance in natural vision: Reinterpreting salience. Journal of Vision, 11(5), 1-23.

Watson, M. R., Brennan, A. A., Kingstone, A., \& Enns, J. T. (2010). Looking versus seeing: Strategies alter eye movements during visual search. Psychonomic Bulletin and Review, 17(4), 543-549.

Yates, D. J., \& Stafford, T. (2011). Insights into the function and mechanism of saccadic decision making from targets scaled by an estimate of the cortical magnification factor. Cognitive Computation, 3(1), 89-93.

Zelinsky, G. J., \& Sheinberg, D. L. (1997). Eye movements during parallel-serial visual search. Journal of Experimental Psychology: Human Perception and Performance, 23(1), 244-262. 